\title{
Quantitative Analyses of Skin Cancer Research in Pakistan
}

\author{
Syeda Hajrah Rehman', Bushra Majid², \\ Sofia Ali Syed ${ }^{3}$, Muhammad Asif Qureshi ${ }^{4}$
}

\section{Summary}

In order to investigate the current status of skin cancer research output in Pakistan, International (PubMed) and national (PakMediNet) scientific databases were searched using variety of keywords to retrieve relevant publications. A strict inclusion criterion was applied to select skin cancer publications for final analyses. Data were recorded by two authors and consistent data were entered into SPSS and Microsoft Excel and analyzed for annual growth rate and frequencies. Of 116 articles that were finally included in the study, 74 were original articles, 24 were case-reports, 10 were review articles, three were editorials, two were research communications and one each of case-series, correspondence and response to letter to the editor. The first article on skin cancer from Pakistan was published in 1976 whereas the last article included in our study was published in December 2018. Excluding Karachi, most of the cities have no contribution in the field of skin cancer. Since 1976 to date, the average number of publications per year has been low, with only 2.7 publications per year. Skin cancer research is alarmingly scarce in Pakistan. This calls for immediate attention by all concerned to contribute and devise appropriate measures towards skin cancer research in Pakistan.

KEYWORDS: Skin cancer, Research output, International literature, Pakistan.

How to cite this:

doi: https://doi.org/10.12669/pjms.37.2.2889

Rehman SH, Majid B, Syed SA, Qureshi MA. Quantitative Analyses of Skin Cancer Research in Pakistan. Pak J Med Sci. 2021;37(2):582-587. doi: https://doi.org/10.12669/pjms.37.2.2889

This is an Open Access article distributed under the terms of the Creative Commons Attribution License (http://creativecommons.org/licenses/by/3.0), which permits unrestricted use, distribution, and reproduction in any medium, provided the original work is properly cited.

1. Syeda Hajrah Rehman, MBBS Student. Dow Medical College,

2. Bushra Majid, MBBS Student.

Dow Medical College,

3. Sofia Ali Syed, BDS, M.Phil.

Associate Professor,

Dow Dental College,

4. Muhammad Asif Qureshi, MBBS, PhD, Postdoc (Germany), M.A. Professor of Pathology, Dow International Medical College,

1-4: Dow University of Health Sciences,

Karachi, Pakistan.

Correspondence:

Dr. Muhammad Asif Qureshi, MBBS, PhD, Postdoc (Germany), M.A. Professor of Pathology,

Dow International Medical College,

Dow University of Health Sciences,

Karachi, Pakistan.

Email: asif@asifqureshi.com

* Received for Publication:

* Revision Received:

* Revision Accepted:
May 12, 2020

November 30, 2020

December 6, 2020

\section{INTRODUCTION}

Skin cancer is the fifth commonest cancer across the globe accounting for approximately $5.8 \%$ of cases (melanoma being 1.6\%) and an overall mortality of 0.6 to $0.7 \%{ }^{1}$ In Pakistan, while there are no national level cancer registration system, regional cancer registries like Punjab Cancer Registry (PCR) and others have reported high incidence of skin cancers in contrast to Globocan 2018 report which lists non melanoma skin cancers (NMSC) as $18^{\text {th }}$ and melanoma as $32^{\text {nd }}$ most common cancer of Pakistan., ${ }^{2,3}$ According to the PCR, skin cancer ranks as the eighth and ninth most common cancer in females and males respectively in 2017. ${ }^{4}$ Moreover, Karachi Cancer Registry (KCR) also reported skin cancer amongst the top eight cancers in females in Karachi..$^{5}$ Recently, a pathology-based cancer registry of Dow University of Health Sciences 
(DUHS) listed NMSC, as the fifth most commonly diagnosed cancer (being second most common in males and fifth most common in females), suggesting a substantial increase of skin cancer burden in Karachi. ${ }^{6}$ Reported cases in Karachi and Larkana are far common than in other parts of Pakistan (like Hazara-district) proving the wellestablished fact that there exists an inverse relation between skin cancer incidence and distance from the equator. ${ }^{7}$

Taken together, these data suggest an increase of skin cancer throughout Pakistan in recent

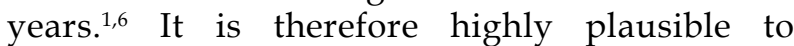
undertake appropriate measures to address this cancer type. One such step could be to identify if Pakistani scientists are investigating this cancer appropriately to identify novel molecules of pathogenic, diagnostic, therapeutic and prognostic significance. To the best of our knowledge, there are no systematic analyses available to delineate the current status of skin cancer research in Pakistan.

In the research described herein, we quantitatively investigated the current status of skin research output in Pakistan. The objectives of this study were (a) to investigate the total skin cancer research output to date originating from Pakistan, (b) to investigate the types/categories and research foci of skin cancer research related publications published from Pakistan, and (c) to investigate major contributing universities/ research centres and cities towards skin cancer research output from Pakistan.

\section{METHODS}

This observational study was conducted at the Dow University of Health Sciences Karachi using a three-step inclusion criteria stratified approach; 1) Literature-search on skin cancer 2) Shortlisting of publications/studies according to inclusion criteria 3) Indexation and analyses of selected studies (Fig.1). Since this study was based on tertiary analyses of publically available data, it does not require ethical/IRB approval.

All duplicate entries were excluded from analyses. Only those publications were included in the study where a) skin cancers in Pakistan was the primary focus of publication or discussed in other ways such as epidemiological studydesigns addressing skin cancer-burden in Pakistan b) studies conducted in Pakistan or their area of concern was skin cancer in Pakistan c) at least first author was affiliated to any institution of Pakistan.
Details of the steps undertaken in the analyses described herein are described as follows; Literature search was performed by two authors. In order to retrieve skin cancer related literature in Pakistan, both international (PubMed) and national (PakMediNet) scientific databases were searched irrespective of study design. In order to extract maximum number of literature search, a wide variety of key words including "skin-malignancy and Pakistan", "skin cancer and Pakistan", "squamous-cell-carcinoma and Pakistan", "skin-neoplasia and Pakistan", "basalcell-carcinoma and Pakistan", "melanoma and Pakistan", "skin-carcinoma and Pakistan", "skincancer-epidemiology and Pakistan", "cutaneousmalignancies and Pakistan", "cutaneouscancers and Pakistan", "cutaneous-neoplasia and Pakistan", "skin metastasis and Pakistan" and "cutaneous-carcinoma and Pakistan" were entered. To further strengthen maximum number of literature search, Google and GoogleScholar search engines with restriction to retrieve webpages of Pakistan were used. Moreover, we did not use any database filters.

Two authors indexed the data in MicrosoftExcel (MS) sheets separately to avoid any error. As previously described, ${ }^{8}$ the selected studies were recorded for their PubMed ID, year and type of publication, name of the journal, title and design of study, authors affiliation/institution, research focus, and number of citations. Publications which were not indexed in PubMed (Medline) were included in non-indexed publications. The entered data of both the authors were compared and discrepancies (if found) were removed to generate a final indexed data-sheet. Final data were then entered into SPSS to calculate annual growth rate and descriptive analyses as described in Table-I and Table-II. All descriptive statistical analyses described herein were conducted using SPSS.

\section{RESULTS}

Initially, a total of 176 studies were retrieved. Of these, 16 duplicate entries were removed leaving a total of 160 studies were further analysed. Of 160 studies, 44 did not meet the inclusion criteria, and were removed from our study. The remaining 116 studies were subjected to final analyses described herein (Fig.1).

Annual growth of publications: The first and last article on skin cancer from Pakistan was published in 1976 and 2018 respectively. Only 10 studies were published during 24 years since the first publication. 


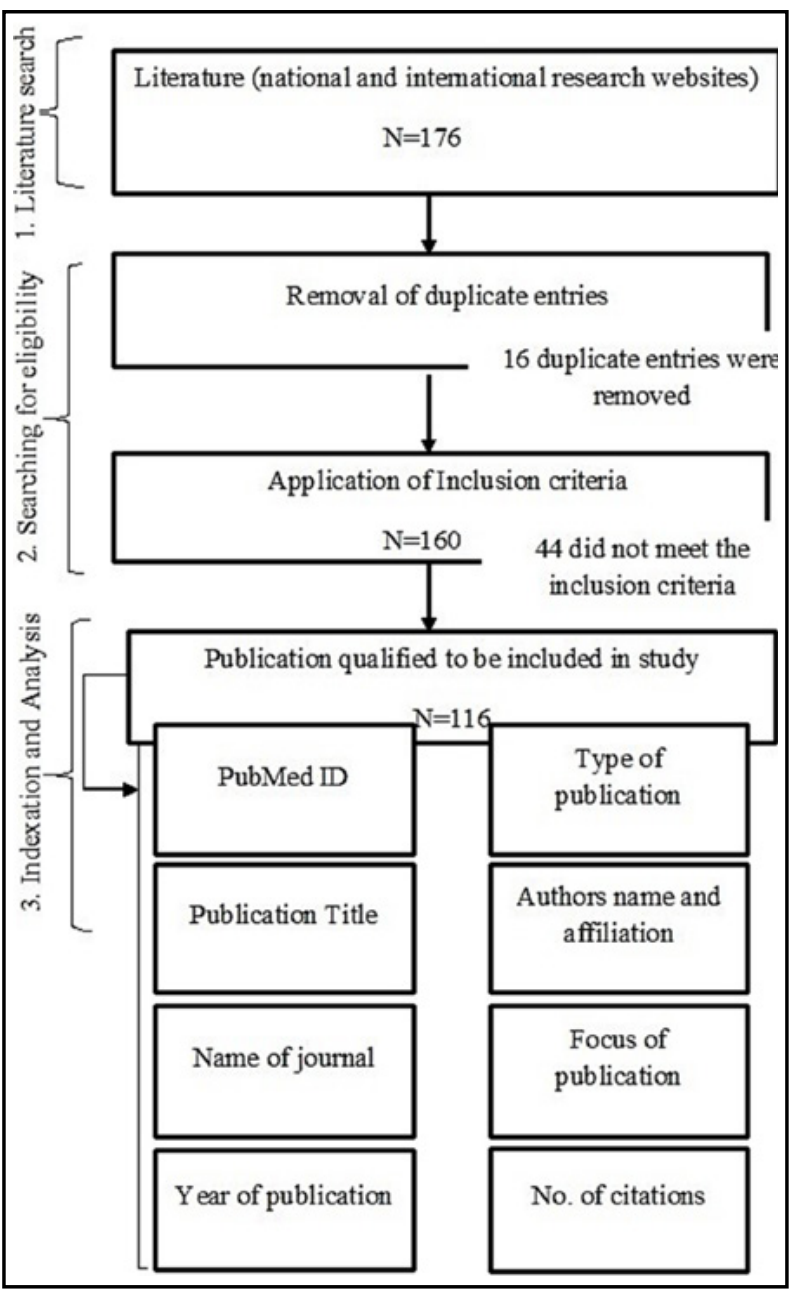

Fig.1: Schematic Representation of the Working-Algorithm of Study.

A total of 16 articles were published during 20002005 (annual growth rate of $9.96 \%$ ). Within the next five years (2006-2010), 26 articles were published

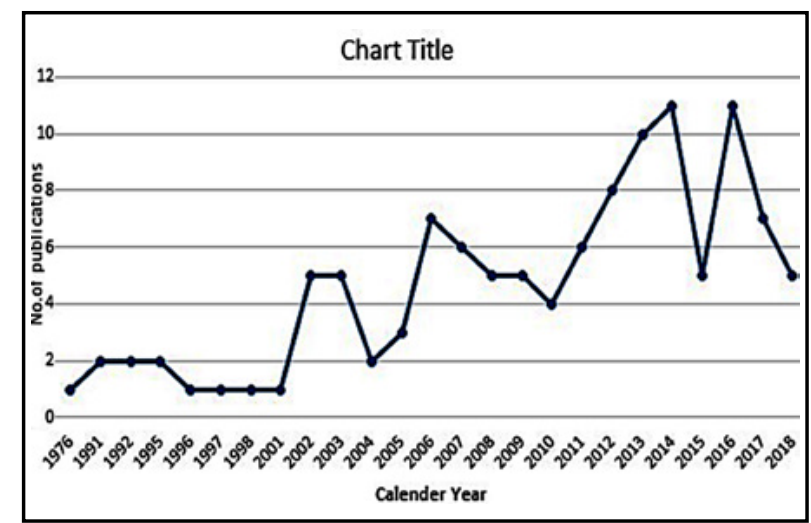

Fig.2: Annual Growth of Skin Cancer Related Publications from Pakistan since 1976 to date.

(annual growth rate of 12.5\%). During 2011-2015, a total of 40 articles were published (annual growth rate of $10.76 \%$ ). From 2016 to 2018, 24 articles were published (annual growth rate of $-13.3 \%$ ) with 2016 being the year with highest number of published articles $(n=12)$. Since 1976 to date, the average number of publications per year has been low, with only 2.7 publications per year (Fig.2)

Types and research foci of publications: Of the 116 articles, $81 \%(\mathrm{n}=94)$ were published in indexed (PubMed) journals and 19\% $(n=22)$ were published in non-indexed journals. Table-I represents the major journals (journals with more than two publications were listed) which published the skin cancer research from Pakistan. Of the 116 publications, $63.7 \%(\mathrm{n}=74)$ were original-articles, $20.6 \%(n=24)$ were case-reports, $8.6 \%(n=10)$ were review-articles, three were editorials, two were research-communications and one each of caseseries, correspondence and response to letter to the editor. Of the 74 original-articles, $28.3 \%(n=21)$

Table-I: Major Journals to publish skin cancer research from Pakistan.

\begin{tabular}{lcccc}
\hline Name of journals & No. of publications $n(\%)$ & PubMed indexed & Impact factor & H-index \\
\hline J Pak Med Assoc & $10(8.62 \%)$ & Yes & 0.718 \\
Ann Med Surg & $7(6.03 \%)$ & Yes & - \\
Asian Pac J Cancer Prev & $7(6.03 \%)$ & Yes & - \\
J Coll Physicians Surg Pak & $6(5.17 \%)$ & Yes & 0.439 \\
Skin Res Technol & $5(4.31 \%)$ & Yes & 1.489 \\
J Pak Assoc Derma & $4(3.44 \%)$ & No & - \\
J Cutan Pathol & $3(2.58 \%)$ & Yes & - \\
Pak Armed Forces Med J & $3(2.58 \%)$ & No & - \\
\hline
\end{tabular}

* journals with more than 2 publications are included. 
Syeda Hajrah Rehman et al.

Table-II: Major Contributing Institutions to Skin Cancer Research in Pakistan.

\begin{tabular}{lll}
\hline University/Research Institutions & City & $N(\%)$ \\
\hline Agha Khan University Hospital & Karachi & $11(9.48 \%)$ \\
Dow University of Health Sciences & Karachi & $7(6.03 \%)$ \\
Armed Forces Institute of Pathology & Rawalpindi & $5(4.31 \%)$ \\
Pakistan Institute of Medical Sciences & Islamabad & $5(4.31 \%)$ \\
Quaid-e-Azam University & Islamabad & $5(4.31 \%)$ \\
King Edward Medical University & Lahore & $4(3.44 \%)$ \\
National Textile University & Faisalabad & $4(3.44 \%)$ \\
COMSATS Institute of Information Technology & Wah & $3(2.58 \%)$ \\
Institute of Radiotherapy and Nuclear Medicine (IRNUM) & Peshawar & $3(2.58 \%)$ \\
Sindh Institute of Urology and Transplantation (SIUT) & Karachi & $3(2.58 \%)$ \\
\hline
\end{tabular}

were based on the prevalence and occurrence of the skin cancer, $14.8 \%(\mathrm{n}=11)$ publications were hospital based, $8.1 \%(n=6)$ were pathology based, four population based, $14.8 \%(n=11)$ were centred on the diagnostic modalities for skin cancer, $10.8 \%(n=8)$ studies were laboratory-based, $12.2 \%$ $(n=9)$ focused on therapeutic-interventions for various skin malignancies and $2.7 \%(n=2)$ were comparisons. Of 24 case-reports, $25 \% \quad(n=6)$ comprised benign yet unique presentations of cutaneous-tumours while $75 \% \quad(n=18)$ discussed the malignant-tumours presenting at a rare site or as a rare occurrence while one case reported skin malignancy as part of Gorlin-syndrome.

Of the 10 reviews, $40 \% \quad(n=4)$ discussed the aetiology and diagnosis of skin malignancies, 20\% $(n=2)$ focused on the role of anticancer agents for various malignancies including the skin cancer and $20 \%(n=2)$ focused on the management, $10 \%$ $(n=1)$ focused on the protective factor for skin cancer while one reviewed the mechanism of melanomagenesis.

Of two research-communications, one compared the clinical features/outcome of patients with nodal or extra-nodal nonHodgkin-lymphoma while the other one focused on epidemiology of basal-cell-carcinoma and importance of clearing excision margins of the malignancy. The three editorials highlighted the importance of cancer registration, case of nodular-melanoma and its management and role of flavonoid in skin cancer.

Thecase-series focused on malignant-cutaneousadnexal-tumour while the correspondence focused on various features of melanocytic-nevi across diverse geographical locations.

Authors affiliations and contributing institutions: A total of 54 Pakistani institutions/ research centres contributed in publications. Of these, $67 \%(\mathrm{n}=34)$ were from public/government set-up, $29.6 \%(n=16)$ were from private setups. Leading institute was Agha Khan University Hospital (AKUH), Karachi. Interestingly, these 54 institutions were only from the 19 cities of Pakistan with Karachi being the main contributor. The analysis thus revealed that most of the cities of Pakistan have no contribution in the field of skin cancer (Table-II)

Number of citations received: Of 116 articles, a total of $13.7 \%(n=16)$ articles did not receive any citation. Of these, $25 \%(n=4)$ and $75 \%(n=12)$ articles were published in indexed and nonindexed journals respectively. The remaining 100 articles were cited at least once and therefore summed-up to a total of 1886 citations. Of these, $77.4 \%(\mathrm{n}=1460)$ citations belonged to the originalarticles under various sub-categories namely therapeutic, diagnostic, genetics, hospital/ laboratory, histopathology, clinicopathological and comparison based. Of 1460 citations, a total of $30 \%(n=432)$ citations were received by laboratory-based studies which by large showed the maximum number of citations in skin cancer research.

\section{DISCUSSION}

With a substantial rise in skin cancer burden in Pakistan, it is highly plausible to investigate if the 
rise is paralleled by relevant research. This report, to the best of our knowledge is the first report that systematically present all data available with reference to skin cancers in Pakistan. We report extreme paucity of skin cancer research in Pakistan reflecting a generalized neglect towards healthcare research as also highlighted in the National Health Plan. ${ }^{9}$

Most of the studies were related to frequency of skin cancer at regional level with paucity of national level data. Moreover, only 2.6 publications/year indicates skin cancer research in Pakistan as an un-attended issue. Alarmingly, majority of the skin cancer research published from Pakistan was not published in indexed journals, leading to low visibility and citation of these reports. ${ }^{10,11}$

Of 116 studies that we investigated, only 74 were original-articles while 21 of them were based on the prevalence/frequencies of skin cancer. Only three studies discussed post-transplant skin malignancies which are documented as common malignancies and common cause of mortality in transplant recipients. ${ }^{12}$ Sindh Institute of Urology and Transplantation (SIUT), being one of the major transplant centres in Pakistan contributed only two studies related to post-transplant malignancies. The third was a review published by DUHS.

Alarmingly, little investigation has been made to address novel diagnostic/treatment modalities regarding skin cancer research in Pakistan. We did not find any relevant randomized-controlledtrials or meta-analysis in this regard. These facts highlight obvious lack of priority to skin cancer research by the researchers as well as relevant policy makers.

Highest number of publications were published from Karachi followed by Islamabad. Private institute with the largest number of publications since 1976 was AKUH with 11 published studies. Of these, four were reviews, three case-reports and three original-articles. No article was published from this institute on skin cancer in 2018. Such little output by a 1203 bedded-hospital is a matter of great concern. ${ }^{13}$ A public-sector university (DUHS) contributed total seven studies. Of these, three were casereports, three were original-articles and one was review-article. However, no article was published for last two years. Lack of funds, research guidelines and proper statistics of skin cancer in the country may have allowed this situation to occur. Moreover, SKMH\&RC (since inception) produced only two publicationsan evidence of negligible performance by its research department.

It is important to mention that Pakistan shares a functionally active border with neighbouring countries (such as Iran and Afghanistan) where skin cancer incidence is high. ${ }^{1}$ There was only one study from Pakistan describing skin cancer as the $2^{\text {nd }}$ most common malignancy in Afghan refugee population. ${ }^{14}$ Since then, number of refugees, sociodemographics and cultural practices have changed. It is therefore highly relevant for researchers in those areas to conduct follow up studies to delineate current situation of skin cancer in those areas. These data suggest the dire need for setting-up a national cancer registry that can ease the direction of decisionmakers and future researchers. ${ }^{15}$ Taken together, we highlight serious paucity of skin cancer research in Pakistan requiring immediate attention by all relevant stakeholders.

Limitations of the study: While the study effectively highlights status of skin cancer research in Pakistan, it is, by definition of the inclusion criteria described herein, unavoidably possible that articles are not listed in PubMed and/or PakMediNet are missed in the analyses described herein.

\section{CONCLUSION}

Skin cancer research is alarmingly scarce in Pakistan. This calls for immediate attention by health policy-makers, institutions as well as researchers to contribute and devise appropriate measures towards skin cancer research in Pakistan.

\section{Source of funding: None.}

Conflict of Interest: None.

\section{REFERENCES}

1. Bray F, Ferlay J, Soerjomataram I, Siegel RL, Torre LA, Jemal A, et al. Global cancer statistics 2018: GLOBOCAN estimates of incidence and mortality worldwide for 36 cancers in 185 countries. CA Cancer J Clin. 2018;68(6):394424. doi: 10.3322/caac. 21492

2. Shaukat Khanam Memorial Trust. Annual Cancer Registry Report-2018 of the Shaukat Khanam Memorial Cancer Hospital \& Research Center [Internet]. Lahore: Shaukat Khanum Cancer Registry; 2019 Website: [https://shaukatkhanum.org.pk/wpcontent/uploads/2019/07/acrr-2018.pdf] Retrieved on Sep 24, 2019. 
3. Ferlay J, Colombet M, Soerjomataram I, Mathers C, Parkin DM, Pineros M, et al. Estimating the global cancer incidence and mortality in 2018: GLOBOCAN sources and methods. Int J Cancer. 2019;144(8):1941-1953. doi: 10.1002/ijc.31937

4. Punjab Cancer Registry. Punjab Cancer Registry Report-2017 [Internet]. Lahore: Punjab Cancer Registry; 2018. Website:[http://punjabcancerregistry.org.pk/ reports/PCR_2017.pdf] Retrieved on Sep 24, 2018.

5. Bhurgri Y. Karachi Cancer Registry Data-implications for the National Cancer Control Program of Pakistan. Asian Pac J Cancer Prev. 2004;5(1):77-82.

6. Qureshi MA, Khan S, Sharafat S, Quraishy MS. Common cancers in Karachi, Pakistan: 2010-2019 Cancer Data from the Dow Cancer Registry. Pak J Med Sci. 2020;36(7):15721578. doi: 10.12669 / pims.36.7.3056

7. Ahmed A, Alam MB, Khan W, Badar A, Shah SH. Frequency and characteristics of skin cancers diagnosed at Ayub Medical College, Abbottabad Pakistan from 1995-2003. J Ayub Med Coll Abbottabad. 2007;19(4):3-6.

8. Khan Z, Muller S, Ahmed S, Tonnies J, Nadir F, Zeeb SF. Quantitative Review of Oral Cancer Research Output from Pakistan. Asian Pac J Cancer Prev. 2015;16(11):4733-4739.

9. Government of Pakistan. Pakistan Health Policy 2010-2015 [Internet]. 2009 July. Website:[https://www.uhc2030. org/fileadmin/uploads/ihp/Documents / Country Pages/Pakistan/PakistanHealthPolicy2010-2015.pdf] Retrieved on Sep 24, 2019.

10. Vines TH, Andrew RL, Bock DG, Franklin MT, Gilbert KJ Kane NC, et al. Mandated data archiving greatly improves access to research data. FASEB J. 2013;27(4):1304-1308. doi: $10.1096 /$ fj.12-218164

11. Akhigbe RE. Scientific journals: Indexation and impact factor. Lung India. 2012;29(3):300-301.
12. Mittal A, Colegio OR. Skin cancers in organ transplant recipients. Am J Transplant. 2017;17(10):2509-2530. doi: 10.1111/ajt.14382

13. The Agha Khan University. The Agha Khan University Report-2017 Website:[https://www.aku.edu/about/ report/Documents/report-2017.pdf] Retrieved on Sept 16, 2019.

14. Khan SM, Gillani J, Nasreen S, Zai S. Cancer in north west Pakistan and Afghan refugees. J Pak Med Assoc. 1997;47(4):122-124.

15. World Health Organization. National Cancer Control Programmes: policies and managerial guidelines 2002 Geneva: WHO;2002 Website: [https://apps.who.int/ iris/bitstream/handle/10665/42494/9241545577. pdf? sequence $=1 \&$ isAllowed=y] Retrieved on Sep 24, 2018.

\section{Author's Contribution:}

SHR: Data retrieval, indexation, and manuscript drafting.

BM: Data retrieval, indexation and manuscript drafting.

SAS: Facilitation and monitoring of data analyses and manuscript drafting.

MAQ: inception of idea, execution and management of the project. Data indexation, supervision of data analyses and is responsible for integrity of results described herein.

All authors read and agreed to the final version of the manuscript. 